\title{
Correction: Hippocampal neural stem cells and microglia response to experimental inflammatory bowel disease (IBD)
}

loannis-Alexandros Gampierakis • Yassemi Koutmani - Maria Semitekolou • loannis Morianos • Alexia Polissidis • Antonia Katsouda · Ioannis Charalampopoulos • Georgina Xanthou • Achille Gravanis • Katia P. Karalis

Published online: 1 September 2020

(c) Springer Nature Limited 2020

Correction to: Molecular Psychiatry

https://doi.org/10.1038/s41380-020-0651-6

published online 22 January 2020

In the original version of this article, the authors "Alexia Polissidis" and "Antonia Katsouda" were omitted from the author list. The author list has now been updated to include these authors, and their contribution to the article has been removed from the acknowledgements. This has been corrected in both the PDF and HTML versions of the article. 